ances in position in some combinations, it is probable that a definite and approximately equal share of space must be accorded to each electron. The approximately rectilinear curve points to this fact. Both properties, however, differ somewhat in different combinations, so that some slight modification is to be understood. It is, however, sufficiently interesting to be able to trace such a relation when it is considered that neither the atomic volumes nor refractions can be directly measured, but are derived constants.

Some of the variations seem to point to variable relations with the ethereal medium. Negative anomalies, for example, are at present incomprehensible, the whole of the atomic refractions of, say, oxygen in $\mathrm{P}(\mathrm{OEt})_{3}$, for example, disappearing entirely. Some of the per saltum changes are equally difficult to explain. The periodic relationship between the elements points rather to a spiral arrangement (understood in a solid sense) ${ }^{2}$ of the electrons than to a series of rings. This arrangement is due to the fact that the spiral is one of the natural modes of motion of the æther and discrete farticles immersed in it.

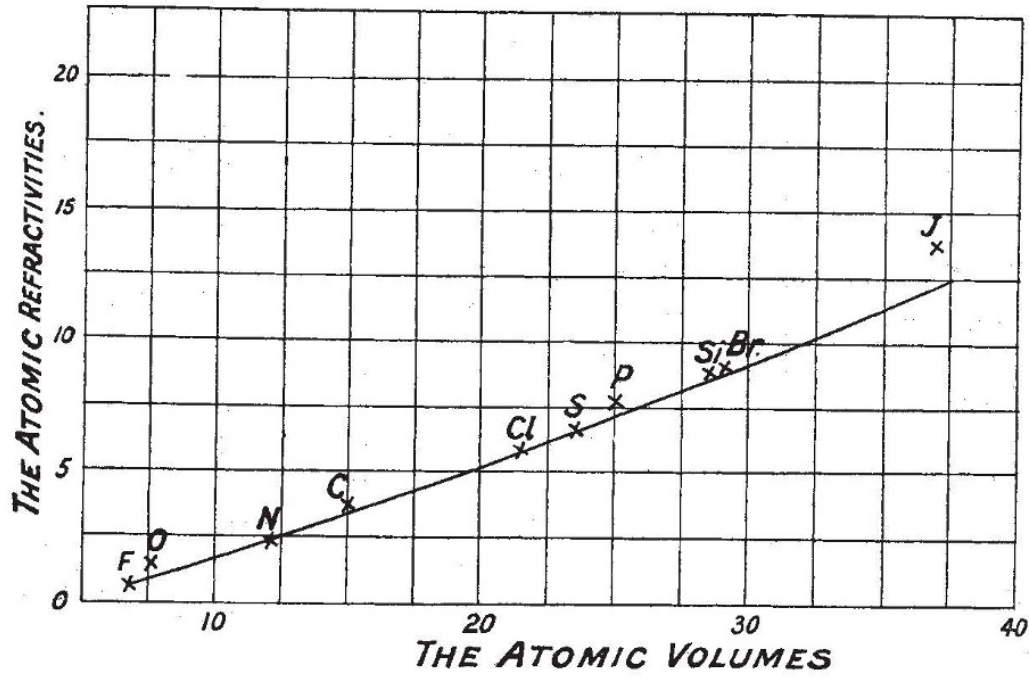

FIC. 1. - A relation between the atomic volumes and the atomic refractivities.

This is shown in the case of spiral nebulæ. The vortex is another condition. Gradual disintegration of the atoms is thus more easily understood, and devolution is reversed evolution.

Up to the present the charge on the electron is regarded as static, but the existence of magnetic properties suggests rotations of electricity on the material particles. This would result in a magnetic flux. It, however, seems to be impossible to distinguish the charges within the atom.

Note.-A numerical relation with the atomic masses suggests itself. $m=3^{r_{a}} \times \mathrm{F}, \mathrm{F}$ being a factor probably representing concentration of matter (atomic density).

$$
m=b_{0} \times \mathrm{F} \quad b_{0} \text { for A:Z. }
$$

$\frac{m}{b_{0}}=F(2,2,3,3)$ not for first series except for C.

$$
\begin{array}{ll}
\mathrm{Cl} & 6 \times 3 \times 2=36 \\
\mathrm{Mn} & 8.8 \times 3 \times 2=52.8(55) \\
\mathrm{Br} & 8.8 \times 3 \times 3=79.2(79.6)
\end{array}
$$

I $13^{\circ} 9 \times 3 \times 3=125^{\circ} 1$ (126).

$F$ diminishes from group 7 io 4 and increases differently in the groups. This relation is, however,

A spherical tourbouillon with a vortical centre and a peripheral rotation (model slip-kıot) based on products, whilst the additive cumulative factor is evident; still, the relation is interesting. The next homologue after $\mathrm{Cl}$ is $\mathrm{Br}$ (red liquid). There is, however, a large gap between the two. Instead of an easily condensible yellow-red vapour at 55 , by some twist we get a metal. There is a similar disparity between $\mathrm{Br}$ and I. Gervaise Le Bas.

6 Springfield Crescent, St. Heliers, Jersey.

\section{Hybridity and the Evolution of Species.}

As the author of the "Theory of Evolution by Means of Hybridisation," I am naturally much interested in the recent papers by Dr. Harrison and Miss Blackburn, which proved beyond any reasonable doubt that most.British rose-species are of hybrid origin, though this was not suspected. The authors based their conclusion that hybridity is one of the prime factors in the evolution of species, if not the only one, on their cytological results, which agree with those of Tackholm on a much larger number of rosespecies from all parts of the world. The reviewer of their papers in NATuRE of September I5, p. 99, does full justice to the importance of these results, and directs attention to Jeffrey's work tending to show that the presence of "bad pollen" is proof of a hybrid origin-a view much strengthened by Brainerd and Petersen's study of the New England Rubi (Vermont Agric. Expt. Sta. Bull. No. 217), in the course of which they find much hybridisation and no forms with entirely good pollen.

To this view the reviewer takes esception. That "bad pollen" is unsafe as a criterion of hybridity is shown, he says, however, by other results. As such he considers the fact that isolated species, such as the Californian Trillium giganteum, the nearest relative of which is in the Eastern States, possess a certain amount of bad pollen. I am sorry to say that I fail to see the bearing of a now isolated habitat on the problem in question. I suppose that the reviewer will agree with me that the origin of Oenothera biennis, which for several centuries has been a feature of the flora of the dunes in many European countries originated elsewhere than in Europe; so why should Trillum giganteum, and Dirca occidentalis and Scoliopus Bigelovii, the other two species with bad pollen which he quotes as proof of his contention, have originated at the spots they now occupy? And if they originated somewhere else, the argument against their possible origin by crossing does not hold good.
Velp, Holland, October 4.
J. P. Lotsy.

IT is perhaps only natural that Dr. Lotsy should take a special view of any facts that bear on his theory of evolution by hybridisation, but in the above letter he is clearly begging the question. In the article on British roses and hybridity to which he refers it was pointed out that the original authors did not consider all British roses to be hybrids, but looked upon the diploid forms and the Pimpinellifoliæ as pure species. In such cases as Trillium, Dirca, and Scoliopus, it is not sufficient for him to suggest that they must be hybrids merely because they have bad pollen. The fact, previously cited, that 